\title{
Addendum to the paper "generalised parabolic sheaves on an integral projective curve"
}

\author{
USHA N BHOSLE \\ School of Mathematics, Tata Institute of Fundamental Research, Homi Bhabha Road, \\ Bombay 400005 , India \\ MS received 21 August 1992
}

In [1] we constructed moduli spaces of generalised parabolic sheaves (GPS in short) of rank $n$ on an integral projective curve, the generalised parabolic structure being at finitely many (smooth) points of the curve. The method of construction was a natural generalisation of the method of Simpson [2] which uses the well-known embedding of Quot scheme into a Grassmannian. The method of [2] was generalised by M S Narasimhan and T R Ramadas to construct moduli of (generalised) parabolic sheaves of rank 2 (on an integral projective curve) with generalised parabolic structure at a point $y$ and usual parabolic structure at finitely many points $y_{j}$ different from $y$ [Appendix, [3]). By oversight the reference to this interesting paper was not given in [1].

We would also like to point out the following correction to $\S 2,[1]$. In $(2-1,[1])$ one should have "a point $q \in \tilde{Q}$ gives for each $j$, a $q_{j}$-dimensional quotient of $\mathbb{C}^{n} \otimes$ $H^{\circ}\left(\mathcal{O}_{D}\right)$, it is given by the composite map $H^{0}\left(\mathcal{O}^{n} \otimes \mathcal{O}_{D}\right) \rightarrow H^{0}\left(\mathscr{F}_{q} \otimes \mathcal{O}_{D_{j}}\right) \rightarrow F_{0}^{j}\left(\mathscr{F}_{q}\right) / F_{1}^{j}\left(\mathscr{F}_{q}\right)$. Hence we get an embedding $Q \rightarrow Z=$ Grass $_{P(m)}\left(\mathbb{C}^{n} \otimes W\right) \times\left(\times_{j} \operatorname{Grass}_{q j}\left(\mathbb{C}^{n} \otimes H^{0}\left(\mathcal{O}_{D_{j}}\right)\right)\right.$. $\ldots$ We denote a point of $Z$ by $\left(P,\left(P_{j}\right)\right)$ where $P: \mathbb{C}^{n} \otimes W \rightarrow U, P_{j}: \mathbb{C}^{n} \otimes H^{0}\left(\mathcal{O}_{D_{j}}\right) \rightarrow U_{j}$ are surjective maps, ...". Also in the expression for $\sigma_{H}$ in Proposition $2 \cdot 2$ and in the proofs of Lemmas $2 \cdot 5$ and $2 \cdot 6$, one should replace " $P_{j}(H)$ " by " $P_{j}\left(H \otimes H^{\circ}\left(\mathcal{O}_{D_{j}}\right)\right)$ ".

\section{References}

[1] Bhosle Usha N, Generalised parabolic sheaves on an integral projective curve, Proc. Indian Acad. Sci. (Math. Sci.), 102 (1992) 13-22

[2] Simpson C T, Moduli of representations of the fundamental group of a smooth projective variety, (Preprint, 1990)

[3] Narasimhan M S and Ramadas T R, Factorisation of generalised theta functions I, T.I.F.R. (preprints 1991-1992) 